\title{
Design of Cosine-Sine Modulated Filter Banks without DC leakage
}

\author{
Marco Maaß, Huy Phan, and Alfred Mertins \\ Institute for Signal Processing, University of Lübeck \\ D-23562 Lübeck, Germany \\ \{maasz,phan,mertins\}@isip.uni-luebeck.de
}

\begin{abstract}
We propose a new design structure for the $M$ band dual-tree wavelet transform based on the idea of using cosine-sine modulated filter banks. The filter coefficients for the real and imaginary parts of the transform will be computed by using cosine- and sine-modulated filter banks, respectively. In the proposed structure, the zero-delay and maximum-delay matrices calculated for the cosine-modulated filter banks will be extended for sine-modulated filter banks with a few sign changes. Furthermore, the resulting structure can be easily enforced with the constraints so that the prototype is able to produce a pair of DC-leakage-free filter banks. The experimental results confirmed these advantageous properties of our proposed design structure.
\end{abstract}

Index Terms-cosine-sine modulated filter bank; modulated filter bank; $M$-band filter bank; dual-tree wavelet transform; shift invariance; DC leakage

\section{INTRODUCTION}

In the literature, it has been shown that the dual tree complex wavelet-transform (DTCWT) offers better shift-invariance and directional selectivity performance than the normal real-valued discrete wavelet-transform (DWT) [1]. In the DTCWT, the two trees correspond to the real and imaginary parts of the filters. Both trees use real-valued filter coefficients and perform a normal DWT. The DTCWT has been extended to the $M$ band DTCWT [2] under further necessary conditions for the corresponding wavelets to form a Hilbert-transform pair. However, if one subband filter in one tree is an FIR filter, the corresponding filters in the dual tree have to be IIR filters [2] (see Fig. 1). The same properties were earlier shown for the DTCWT [1]. The main difficulty of this structure is to design FIR filters for both trees so that they approximately correspond to a Hilbert-transform wavelet pair. In [3], a simple approach for designing $M$-band DTCWT was proposed by using the dual-tree complex packet transform. Unfortunately, this approach limits the number of bands to $M=2^{r}$ with $r \in \mathbb{N}$.

A more recent approach is to design equivalent $M$-band DTCWT transform by using modulated filter banks [4]. We pursue this approach in this work. However, we only use one prototype filter and modulate it to design all subband filters. The work in [4] shows that using cosine-sine modulated filter banks (CSMFB) (Fig. 1) introduced in [5] can be interpreted as $M$-band DTCWT by using the same prototype for the analysis and synthesis filters. The first real part filter bank is a normal cosine-modulated filter bank (CMFB), while the dual filter bank is a sine-modulated filter bank (SMFB). Other filter bank structures of the modulated filter banks also show to have equivalent properties to the $M$-band DTCWT [6], [7].

We introduce another factorization based on the filter bank structure presented in [8] for CMFB and extend it to SMFB under the assumption of the same prototype for both analysis and synthesis filters. We will show that the factorization of the CMFB and SMFB produces nearly the same coefficients due to the fact that only some signs are necessarily changed. That is, the factorization for the CMFB can be directly used for calculating the following factorization of SMFB. As a result, it is possible to enforce the constraint that the modulated filters will have no DC-leakage behavior.

The rest of the paper is organized as follows: In Section II we briefly introduce the CSMFB and the lattice structure that used in [9] and [10]. Next, in Section III, we explain the new factorization based on zero-delay and maximumdelay matrices of CMFB and show that for the SMFB the same factorization follows directly. After that, in Section IV, we calculate the constraints that enforce the factorization to have CSMFB without DC-leakage. Finally, in Section V we present experimental results in which enforcing the DCleakage constraints does not influence the behavior of the filter bank too much.

We use the following notation throughout our formulations:

- $H(z)$ : the $z$-transform of an impulse response $h(n)$,

- $\operatorname{diag}\left(x_{1}, x_{2}, \ldots, x_{n}\right):$ a diagonal matrix with $x_{1}, x_{2}, \ldots, x_{n}$ on the diagonal,

- $I$ : the identity matrix,

- $\boldsymbol{J}$ : the counter-identity matrix,

- $W_{M}^{l}:=e^{j \frac{2 \pi}{M} l}$,

- $\prod_{j=1}^{v} \boldsymbol{L}_{j}=\boldsymbol{L}_{1} \cdot \boldsymbol{L}_{2} \cdot \ldots \cdot \boldsymbol{L}_{v}$ where the order of matrices is important.

\section{COSINE-SINE MODULATED FILTER BANKS}

A CSMFB has two $M$-channel filter banks, each of which is decimated maximally by $M$, with system functions $H_{k}(z), \tilde{H}_{k}(z), F_{k}(z), \tilde{F}_{k}(z)$, as illustrated in Fig. 1. The filter coefficients can be expressed in form of a given prototype filter 


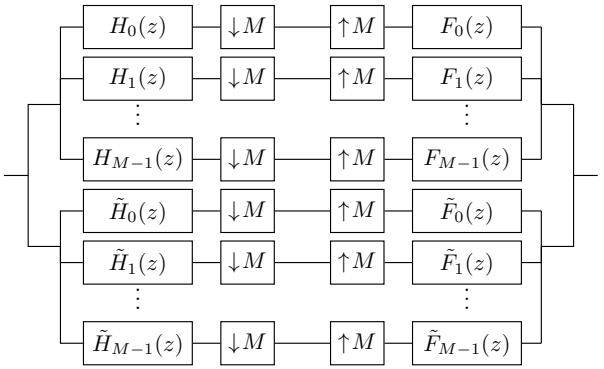

Fig. 1. $M$-band CSMFB and $M$-band DTCWT

$p(n)$ as follows [11]:

$$
\begin{aligned}
& h_{k}(n)=2 p(n) \cos \left(\left(k+\frac{1}{2}\right) \frac{\pi}{M}\left(n-\frac{N-1}{2}\right)+\theta_{k}\right) \\
& \tilde{h}_{k}(n)=2 p(n) \sin \left(\left(k+\frac{1}{2}\right) \frac{\pi}{M}\left(n-\frac{N-1}{2}\right)+\theta_{k}\right) \\
& f_{k}(n)=2 p(n) \cos \left(\left(k+\frac{1}{2}\right) \frac{\pi}{M}\left(n-\frac{N-1}{2}\right)-\theta_{k}\right) \\
& \tilde{f}_{k}(n)=-2 p(n) \sin \left(\left(k+\frac{1}{2}\right) \frac{\pi}{M}\left(n-\frac{N-1}{2}\right)-\theta_{k}\right),
\end{aligned}
$$

where $k \in\{0,1, \ldots, M-1\}, \theta_{k}=(-1)^{k} \frac{\pi}{4}$, and $N$ is the length of $p(n)$. It has been shown that the arising filter bank can be seen as $M$-Band DTCWT [4], where filtering a signal with the filter pair $\left\{H_{k}(z), \tilde{H}_{k}(z)\right\}$ yields the real and imaginary parts of a complex wavelet transform. Furthermore, we can obtain the complex modulated filter banks from CSMFB [12]. Let $\boldsymbol{E}(z)$ and $\tilde{\boldsymbol{E}}(z)$ denote the polyphase matrices of the CMFB and SMFB, respectively. The vectors $\boldsymbol{h}(z)=\left[H_{0}(z), \ldots, H_{M-1}(z)\right]^{T}$ and $\tilde{\boldsymbol{h}}(z)=$ $\left[\tilde{H}_{0}(z), \ldots, \tilde{H}_{M-1}(z)\right]^{T}$ can be obtained in form of the polyphase matrices:

$$
\begin{aligned}
\boldsymbol{h}(z) & =\boldsymbol{E}\left(z^{M}\right)\left[1, z^{-1}, \ldots, z^{-(M-1)}\right]^{T} \\
\tilde{\boldsymbol{h}}(z) & =\tilde{\boldsymbol{E}}\left(z^{M}\right)\left[1, z^{-1}, \ldots, z^{-(M-1)}\right]^{T} .
\end{aligned}
$$

On another hand, the polyphase matrices can be expressed as lattice structures [9]-[11], [13]:

$$
\begin{gathered}
\boldsymbol{E}(z)=\boldsymbol{C}_{\mathrm{IV}} \boldsymbol{W} \boldsymbol{\Lambda}(z) \boldsymbol{D}_{0} \prod_{k=1}^{K-1}\left(\boldsymbol{\Lambda}\left(z^{2}\right) \boldsymbol{D}_{k}\right) \\
\boldsymbol{D}_{k}=\left[\begin{array}{cc}
-\boldsymbol{C}_{k} & \boldsymbol{S}_{k} \boldsymbol{J} \\
\boldsymbol{J} \boldsymbol{S}_{k} & \boldsymbol{J} \boldsymbol{C}_{k} \boldsymbol{J}
\end{array}\right], \boldsymbol{\Lambda}(z)=\left[\begin{array}{cc}
z^{-1} \boldsymbol{I} & \mathbf{0} \\
\mathbf{0} & \boldsymbol{I}
\end{array}\right] \\
\tilde{\boldsymbol{E}}(z)=\boldsymbol{S}_{\mathrm{IV}} \boldsymbol{W} \boldsymbol{\Lambda}(z) \tilde{\boldsymbol{D}}_{0} \prod_{k=1}^{K-1}\left(\boldsymbol{\Lambda}\left(z^{2}\right) \tilde{\boldsymbol{D}}_{k}\right) \\
\tilde{\boldsymbol{D}}_{k}=\left[\begin{array}{cc}
\boldsymbol{C}_{k} & \boldsymbol{S}_{k} \boldsymbol{J} \\
\boldsymbol{J} \boldsymbol{S}_{k} & -\boldsymbol{J} \boldsymbol{C}_{k} \boldsymbol{J}
\end{array}\right]=\left[\begin{array}{cc}
\boldsymbol{I} & \mathbf{0} \\
\mathbf{0} & -\boldsymbol{I}
\end{array}\right] \boldsymbol{D}_{k}\left[\begin{array}{cc}
-\boldsymbol{I} & \mathbf{0} \\
\mathbf{0} & \boldsymbol{I}
\end{array}\right]
\end{gathered}
$$

where $\boldsymbol{C}_{k}=\operatorname{diag}\left(\cos \theta_{k 0}, \ldots, \cos \theta_{k M / 2-1}\right), \quad \boldsymbol{S}_{k}=$ $\operatorname{diag}\left(\sin \theta_{k 0}, \ldots, \sin \theta_{k M / 2-1}\right), \quad \boldsymbol{W}=\left[\begin{array}{ll}\mathbf{0} & \boldsymbol{I} \\ \boldsymbol{I} & \mathbf{0}\end{array}\right]$, and $\boldsymbol{C}_{\mathrm{IV}}$ and $\boldsymbol{S}_{\mathrm{IV}}$ are the type-IV discrete cosine-transform and sinetransform matrices.

\section{THE PROPOSED CSMFB STRUCTURE}

\section{A. The filter bank design}

We propose a new structure for cosine-sine modulated filter banks based on the structure for cosine-modulated filter banks proposed in [8]. We express the prototype $p(n)$ by type-1 polyphase components:

$$
P(z)=\sum_{l=0}^{2 M-1} z^{-l} G_{l}\left(z^{2 M}\right), \quad g_{l}(m)=p(2 m M+l),
$$

with $l \in\{0,1, \ldots, 2 M-1\}$. The overall system delay is obtained as $D=2 s M+2 M-1=N-1$. Furthermore, for the CMFB the perfect reconstruction (PR) constraints are fulfilled for

$$
G_{l}(z) G_{2 M-1-l}(z)+G_{M+l}(z) G_{M-1-l}=\frac{z^{-s}}{2 M},
$$

with $l \in\{0,1, \ldots, M-1\}$ [14]. Hence, if we use the same prototype for both CMFB and SMFB, the PR properties of the CMFB will be inherited in SMFB [5].

In order to analyze the polyphase matrix of the CMFB we can write $\boldsymbol{E}(z)$ as in [8]:

$$
\begin{aligned}
\boldsymbol{E}(z) & =\boldsymbol{C}_{1}\left[\begin{array}{c}
\boldsymbol{g}_{0}\left(-z^{2}\right) \\
z^{-1} \boldsymbol{g}_{1}\left(-z^{2}\right)
\end{array}\right], \\
{\left[\boldsymbol{C}_{1}\right]_{k l} } & =2 \cos \left((k+0.5) \frac{\pi}{M}\left(l-\frac{D}{2}\right)+\theta_{k}\right), \\
\boldsymbol{g}_{0}\left(-z^{2}\right) & =\operatorname{diag}\left(G_{0}\left(-z^{2}\right), \ldots, G_{M-1}\left(-z^{2}\right)\right), \\
\boldsymbol{g}_{1}\left(-z^{2}\right) & =\operatorname{diag}\left(G_{M}\left(-z^{2}\right), \ldots, G_{2 M-1}\left(-z^{2}\right)\right) .
\end{aligned}
$$

Due to the symmetries of the cosine functions, it can be shown that

$$
\begin{aligned}
c_{k, M-1-l}^{(1)} & =\left[\boldsymbol{C}_{1}\right]_{k, M-1-l}=(-1)^{s}\left[\boldsymbol{C}_{1}\right]_{k, l} \\
c_{k, l+M}^{(1)} & =\left[\boldsymbol{C}_{1}\right]_{k, l+M}=(-1)^{s-1}\left[\boldsymbol{C}_{1}\right]_{k, 2 M-1-l} .
\end{aligned}
$$

By dividing the polyphase matrix in (7) into the subsystem matrices $\boldsymbol{E}_{l}(z)$ containing the columns of $\boldsymbol{E}(z)$, which are connected over the properties of the modulation matrix $C_{1}$ given by (8), we can write the sub-matrices as

$$
\begin{aligned}
& \boldsymbol{E}_{l}(z)=\left[\begin{array}{cc}
c_{0, l}^{(1)} & c_{0,2 M-1-l}^{(1)} \\
c_{1, l}^{(1)} & c_{1,2 M-1-l}^{(1)} \\
\vdots & \vdots \\
c_{M-1, l}^{(1)} & c_{M-1,2 M-1-l}^{(1)}
\end{array}\right] \boldsymbol{G}_{l}(z), \\
& \boldsymbol{G}_{l}(z)=\left[\begin{array}{cc}
G_{l}\left(-z^{2}\right) & (-1)^{s} G_{M-1-l}\left(-z^{2}\right) \\
(-1)^{s-1} z^{-1} G_{l+M}\left(-z^{2}\right) & z^{-1} G_{2 M-1-l}\left(-z^{2}\right)
\end{array}\right],
\end{aligned}
$$

and the synthesis side of the CMFB polyphase matrix can be expressed as

$$
\boldsymbol{R}(z)=\left[\begin{array}{ll}
z^{-1} \boldsymbol{k}_{1}\left(-z^{2}\right) & \boldsymbol{k}_{0}\left(-z^{2}\right)
\end{array}\right] \boldsymbol{C}_{2}^{t}
$$


where

$$
\begin{array}{r}
{\left[\boldsymbol{C}_{2}\right]_{k, l}=2 \cos \left((k+0.5) \frac{\pi}{M}\left(2 M-1-l-\frac{D}{2}\right)+\theta_{k}\right),} \\
0 \leq l \leq 2 M-1
\end{array}
$$

and

$$
\begin{aligned}
& \boldsymbol{k}_{0}\left(-z^{2}\right)=\operatorname{diag}\left(G_{M-1}\left(-z^{2}\right), \ldots, G_{0}\left(-z^{2}\right)\right), \\
& \boldsymbol{k}_{1}\left(-z^{2}\right)=\operatorname{diag}\left(G_{2 M-1}\left(-z^{2}\right), \ldots, G_{M}\left(-z^{2}\right)\right) .
\end{aligned}
$$

Finally, we obtain the subsystem $\boldsymbol{R}_{l}$ as

$$
\begin{aligned}
\boldsymbol{R}_{l}(z) & =\boldsymbol{K}_{l}(z)\left[\begin{array}{ccc}
c_{0, l}^{(2)} & \cdots & c_{M-1, l}^{(2)} \\
c_{0,2 M-1-l}^{(2)} & \cdots & c_{M-1,2 M-1-l}^{(2)}
\end{array}\right] \\
\boldsymbol{K}_{l}(z) & =\left[\begin{array}{cc}
z^{-1} G_{2 M-1-l}\left(-z^{2}\right) & (-1)^{s-1} G_{M-1-l}\left(-z^{2}\right) \\
(-1)^{s} z^{-1} G_{l+M}\left(-z^{2}\right) & G_{l}\left(-z^{2}\right)
\end{array}\right]
\end{aligned}
$$

and $c_{k, l}^{(2)}=\left[\boldsymbol{C}_{2}\right]_{k, l}$. If the prototype holds the PR condition in (6), the system

$$
\boldsymbol{K}_{l}(z) \boldsymbol{G}_{l}(z)=\frac{(-1)^{-1} z^{-2 s-1}}{2 M} \boldsymbol{I}_{2}, \quad 0 \leq l \leq M-1
$$

also does so [8]. It turns out that if the CMFB fulfills the PR condition, the SMFB also yields PR. As a result, we only need to compute the factorization for the SMFB analysis polyphase matrix. Similar to (7), we can also present the SMFB as

$$
\begin{aligned}
\tilde{\boldsymbol{E}}(z) & =\boldsymbol{S}_{1}\left[\begin{array}{c}
\boldsymbol{g}_{0}\left(-z^{2}\right) \\
z^{-1} \boldsymbol{g}_{1}\left(-z^{2}\right)
\end{array}\right], \\
{\left[\boldsymbol{S}_{1}\right]_{k l} } & =2 \sin \left((k+0.5) \frac{\pi}{M}\left(l-\frac{D}{2}\right)+\theta_{k}\right) .
\end{aligned}
$$

Due to the symmetries of the sine function, we obtain

$$
\begin{aligned}
& s_{k, M-1-l}^{(1)}=\left[\boldsymbol{S}_{1}\right]_{k, M-1-l}=(-1)^{s-1}\left[\boldsymbol{S}_{1}\right]_{k, l}, \\
& s_{k, l+M}^{(1)}=\left[\boldsymbol{S}_{1}\right]_{k, l+M}=(-1)^{s}\left[\boldsymbol{S}_{1}\right]_{k, 2 M-1-l},
\end{aligned}
$$

and the subpolyphase analysis matrix of the SMFB reads

$$
\begin{aligned}
& \tilde{\boldsymbol{E}}_{l}(z)= {\left[\begin{array}{cc}
s_{0, l}^{(1)} & s_{0,2 M-1-l}^{(1)} \\
s_{1, l}^{(1)} & s_{1,2 M-1-l}^{(1)} \\
\vdots & \vdots \\
s_{M-1, l}^{(1)} & s_{M-1,2 M-1-l}^{(1)}
\end{array}\right] \tilde{\boldsymbol{G}}_{l}(z), } \\
& \tilde{\boldsymbol{G}}_{l}(z)=\left[\begin{array}{cc}
G_{l}\left(-z^{2}\right) & (-1)^{s-1} G_{M-1-l}\left(-z^{2}\right) \\
(-1)^{s} z^{-1} G_{l+M}\left(-z^{2}\right) & z^{-1} G_{2 M-1-l}\left(-z^{2}\right)
\end{array}\right] .
\end{aligned}
$$

\section{B. Factorization in zero-delay matrices and maximum-delay matrices}

Since the polyphase filters satisfy the PR constraints in (12) [8], for $\boldsymbol{G}_{l}(z)$ in (9) and $\boldsymbol{K}_{l}(z)$ in (11), the following decompositions always exist:

$$
\begin{aligned}
& \boldsymbol{G}_{l}(z)=\prod_{j=1}^{j_{0}} \boldsymbol{D}_{l, j}(z) \prod_{i=1}^{i_{0}} \boldsymbol{B}_{l, i}(z) \boldsymbol{G}_{l, \text { ini }}(z), \\
& \boldsymbol{K}_{l}(z)=\boldsymbol{K}_{l, \text { ini }}(z) \prod_{i=i_{0}}^{1} \boldsymbol{B}_{l, i}^{-1}(z) \prod_{j=j_{0}}^{1}\left(z^{-\delta_{l, j}-1} \boldsymbol{D}_{l, j}^{-1}(z)\right),
\end{aligned}
$$

where $j_{0}$ can be calculated from $s=0.5 \sum_{j=1}^{j_{0}}\left(\delta_{l, j}+1\right)$ for a fixed value $s$. The zero-delay matrices are defined as

$$
\begin{aligned}
\boldsymbol{B}_{l, i}(z) & =\left[\begin{array}{cc}
0 & 1 \\
1 & b_{l, i} z^{-\beta_{l}, i}
\end{array}\right], \\
\boldsymbol{B}_{l, i}^{-1}(z) & =\left[\begin{array}{cc}
-b_{l, i} z^{-\beta_{l}, i} & 1 \\
1 & 0
\end{array}\right] .
\end{aligned}
$$

The maximum-delay matrices are defined as

$$
\begin{aligned}
\boldsymbol{D}_{l, j}(z) & =\left[\begin{array}{cc}
d_{l, j} & z^{-1} \\
z^{-\delta_{l, j}} & 0
\end{array}\right], \\
z^{-\delta_{l, j}-1} \boldsymbol{D}_{l, j}^{-1}(z) & =\left[\begin{array}{cc}
0 & z^{-1} \\
z^{-\delta_{l, j}} & -d_{l, j}
\end{array}\right] .
\end{aligned}
$$

Finally, the initialization matrices are given by

$$
\begin{aligned}
\boldsymbol{G}_{l, \text { ini }}(z) & =\left[\begin{array}{cc}
g_{l, 0} & g_{l, 1} \\
z^{-1} g_{l, 2} & z^{-1} g_{l, 3}
\end{array}\right], \\
\boldsymbol{K}_{l, \text { ini }}(z) & =\frac{1}{2 M} \cdot \frac{(-1)^{s}}{g_{l, 0} g_{l, 3}-g_{l, 1} g_{l, 2}}\left[\begin{array}{cc}
g_{l, 3} z^{-1} & -g_{l, 1} \\
-g_{l, 2} z^{-1} & g_{l, 0}
\end{array}\right] .
\end{aligned}
$$

Note that the variables $b_{l, i}, d_{l, j}, g_{l, 0}, g_{l, 2}, g_{l, 3}$ are real-valued and $\beta_{l, i}, \delta_{l, j}$ are positive integer numbers.

In case of SMFB, it is also possible to calculate the decomposition in terms of zero-delay, maximum-delay and initialization matrices given the analysis of the polyphase submatrices $\tilde{G}_{l}$ in (15). Therefore, (15) can be re-written as

$$
\tilde{\boldsymbol{G}}_{l}(z)=\prod_{j=1}^{j_{0}} \tilde{\boldsymbol{D}}_{l, j}(z) \prod_{i=1}^{i_{0}} \tilde{\boldsymbol{B}}_{l, i}(z) \tilde{\boldsymbol{G}}_{l, \text { ini }}(z)
$$

with the same definitions of zero-delay, maximum-delay, and initialization matrices as above.

We will show that

$$
\tilde{b}_{l, i}=-b_{l, i}, \quad \tilde{d}_{l, j}=-d_{l, j}, \tilde{\beta}_{l, i}=\beta_{l, i}, \tilde{\delta}_{l, j}=\delta_{l, j}
$$

and that

$$
\tilde{\boldsymbol{G}}_{l, \text { ini }}(z)=(-1)^{j_{0}+i_{0}}\left[\begin{array}{cc}
g_{l, 0} & -g_{l, 1} \\
-z^{-1} g_{l, 2} & z^{-1} g_{l, 3}
\end{array}\right] .
$$

\section{Proof for maximum-delay matrices}

For the sake of brevity, we will omit the argument $z$ in $\boldsymbol{G}_{l}^{j}(z), \tilde{\boldsymbol{G}}_{l}^{j}(z), \boldsymbol{D}_{l, j}(z), \tilde{\boldsymbol{D}}_{l, j}(z), G_{k, l}^{j}(z), \tilde{G}_{k, l}^{j}(z)$ in the next steps. One decomposition step can be accomplished by

$$
\begin{array}{ll}
\boldsymbol{G}_{l}^{j}=\boldsymbol{D}_{l, j}^{-1} \boldsymbol{G}_{l}^{j-1}, & \boldsymbol{G}_{l}^{0}=\boldsymbol{G}_{l} \\
\tilde{\boldsymbol{G}}_{l}^{j}=\tilde{\boldsymbol{D}}_{l, j}^{-1} \tilde{\boldsymbol{G}}_{l}^{j-1}, & \tilde{\boldsymbol{G}}_{l}^{0}=\tilde{\boldsymbol{G}}_{l} .
\end{array}
$$


After $j$ decomposition steps, $\boldsymbol{G}_{l}^{j}$ and $\tilde{\boldsymbol{G}}_{l}^{j}$ have the forms

$$
\begin{aligned}
& \boldsymbol{G}_{l}^{j}=\left[\begin{array}{ll}
G_{0,0}^{j} & G_{0,1}^{j} \\
G_{1,0}^{j} & G_{1,1}^{j}
\end{array}\right] \\
& \tilde{G}_{l}^{j}=\left[\begin{array}{ll}
\tilde{G}_{0,0}^{j} & \tilde{G}_{0,1}^{j} \\
\tilde{G}_{1,0}^{j} & \tilde{G}_{1,1}^{j}
\end{array}\right]=(-1)^{j}\left[\begin{array}{cc}
G_{0,0}^{j} & -G_{0,1}^{j} \\
-G_{1,0}^{j} & G_{1,1}^{j}
\end{array}\right] .
\end{aligned}
$$

It is easy to check that the initial conditions (9) and (15) will be held for $j=0$. As in [8], the factorization has the form

$$
\boldsymbol{G}_{l}^{j}=\left[\begin{array}{cc}
z^{\delta_{j}} G_{1,0}^{j-1} & z^{\delta_{j}} G_{1,1}^{j-1} \\
z G_{0,0}^{j-1}-d_{j} z^{\delta_{j}+1} G_{1,0}^{j-1} & z G_{0,1}^{j-1}-d_{j} z^{\delta_{j}+1} G_{1,1}^{j-1}
\end{array}\right],
$$

where $d_{j}=\frac{g_{0,0}^{j-1}(0)}{g_{1,0}^{j-1}\left(\delta_{j}\right)}=\frac{g_{0,1}^{j-1}(0)}{g_{1,1}^{j-1}\left(\delta_{j}\right)}$ with $g_{1,0}^{j-1}\left(\delta_{j}\right) \neq 0$, $g_{1,0}^{j-1}\left(\delta_{j}\right) \neq 0$ and $g_{1,0}^{j-1}(n)=g_{1,1}^{j-1}(n)=0$ for all $n=$ $0,1, \ldots, \delta_{j}-1$. We see that $\delta_{j}=\tilde{\delta}_{j}$ and

$$
\tilde{d}_{j}=\frac{\tilde{g}_{0,0}^{j-1}(0)}{\tilde{g}_{1,0}^{j-1}\left(\delta_{j}\right)}=\frac{-g_{0,0}^{j-1}(0)}{g_{1,0}^{j-1}\left(\delta_{j}\right)}=-d_{j} .
$$

Similarly, from (24), we can calculate $\tilde{G}_{l}^{j}$ from $\tilde{G}_{l}^{j-1}$ :

$$
\begin{aligned}
\tilde{G}_{l}^{j}= & {\left[\begin{array}{cc}
z^{\tilde{\delta}_{j}} \tilde{G}_{1,0}^{j-1} & z^{\tilde{\delta}_{j}} \tilde{G}_{1,1}^{j-1} \\
z \tilde{G}_{0,0}^{j-1}-\tilde{d}_{j} z^{\tilde{\delta}_{j}+1} \tilde{G}_{1,0}^{j-1} & z \tilde{G}_{0,1}^{j-1}-\tilde{d}_{j} z^{\tilde{\delta}_{j}+1} \tilde{G}_{1,1}^{j-1}
\end{array}\right] } \\
= & (-1)^{j} \times \\
& {\left[\begin{array}{cc}
z^{\delta_{j}} G_{1,0}^{j-1} & -z^{\delta_{j}} G_{1,1}^{j-1} \\
-z G_{0,0}^{j-1}+d_{j} z^{\delta_{j}+1} G_{1,0}^{j-1} & z G_{0,1}^{j-1}-d_{j} z^{\delta_{j}+1} G_{1,1}^{j-1}
\end{array}\right] } \\
= & (-1)^{j}\left[\begin{array}{cc}
G_{0,0}^{j} & -G_{0,1}^{j} \\
-G_{1,0}^{j} & G_{1,1}^{j}
\end{array}\right] .
\end{aligned}
$$

\section{Proof for zero-delay matrices}

After the maximum-delay factorization step, our initial matrices have the forms

$$
\begin{aligned}
& \boldsymbol{G}_{l}^{0}=\prod_{j=j_{0}}^{1} \boldsymbol{D}_{j}^{-1} \boldsymbol{G}_{l}=\left[\begin{array}{cc}
G_{0,0}^{0} & G_{0,1}^{0} \\
G_{1,0}^{0} & G_{1,1}^{0}
\end{array}\right], \\
& \tilde{\boldsymbol{G}}_{l}^{0}=\prod_{j=j_{0}}^{1} \tilde{\boldsymbol{D}}_{j}^{-1} \tilde{\boldsymbol{G}}_{l}=(-1)^{j_{0}}\left[\begin{array}{cc}
G_{0,0}^{0} & -G_{0,1}^{0} \\
-G_{1,0}^{0} & G_{1,1}^{0}
\end{array}\right] .
\end{aligned}
$$

The $i$ th decomposition step can be done by

$$
\boldsymbol{G}_{l}^{i}=\boldsymbol{B}_{l, i}^{-1} \boldsymbol{G}_{l}^{i-1}, \quad \tilde{\boldsymbol{G}}_{l}^{i}=\tilde{\boldsymbol{B}}_{l, i}^{-1} \tilde{\boldsymbol{G}}_{l}^{i-1} .
$$

We now show that $G_{l}^{i}$ and $\tilde{G}_{l}^{i}$ have the following forms

$$
\begin{aligned}
& \boldsymbol{G}_{l}^{i}=\left[\begin{array}{ll}
G_{0,0}^{i} & G_{0,1}^{i} \\
G_{1,0}^{i} & G_{1,1}^{i}
\end{array}\right] \\
& \tilde{\boldsymbol{G}}_{l}^{i}=\left[\begin{array}{ll}
\tilde{G}_{0,0}^{i} & \tilde{G}_{0,1}^{i} \\
\tilde{G}_{1,0}^{i} & \tilde{G}_{1,1}^{i}
\end{array}\right]=(-1)^{j_{0}+i}\left[\begin{array}{cc}
G_{0,0}^{i} & -G_{0,1}^{i} \\
-G_{1,0}^{i} & G_{1,1}^{i}
\end{array}\right],
\end{aligned}
$$

and the initial condition (28) is fulfilled. Again, as in [8] the factorization is given by

$$
\boldsymbol{G}_{l}^{i}=\left[\begin{array}{cc}
G_{1,0}^{i-1}-b_{i} z^{-\beta_{i}} G_{0,0}^{i-1} & G_{1,1}^{i-1}-b_{i} z^{-\beta_{i}} G_{0,1}^{i-1} \\
G_{0,0}^{i-1} & G_{0,1}^{i-1}
\end{array}\right]
$$

where

$$
\begin{aligned}
\beta_{i} & =N_{g, 1,0}-N_{g, 0,0}=N_{g, 1,1}-N_{g, 0,1} \\
b_{i} & =\frac{g_{1,0}^{i-1}\left(N_{g, 1,0}-1\right)}{g_{0,0}^{i-1}\left(N_{g, 0,0}-1\right)}=\frac{g_{1,1}^{i-1}\left(N_{g, 1,1}-1\right)}{g_{0,1}^{i-1}\left(N_{g, 0,1}-1\right)} .
\end{aligned}
$$

Here, $N_{g, u, v}$ denotes the length of the filter $g_{u, v}^{i}(n)$. If $\beta_{i}<0$, we set $\beta_{i}=0$ and $b_{i}=0$.

Now we calculate $\tilde{G}_{l}^{j}$ with the same factorization. Because $\tilde{\boldsymbol{G}}_{l}^{j-1}$ is given in (30), the filter lengths have to be equal and we have $\tilde{\beta}_{i}=\beta_{i}$ and

$$
\tilde{b}_{i}=\frac{\tilde{g}_{1,0}^{i-1}\left(N_{g, 1,0}-1\right)}{\tilde{g}_{0,0}^{i-1}\left(N_{g, 0,0}-1\right)}=\frac{-g_{1,0}^{i-1}\left(N_{g, 1,0}-1\right)}{g_{0,0}^{i-1}\left(N_{g, 0,0}-1\right)}=-b_{i} .
$$

If $\beta_{i}<0$ is not given, because $b_{i}=0=-b_{i}$, similar to (27), we have

$$
\begin{aligned}
& \tilde{\boldsymbol{G}}_{l}^{j}=\left[\begin{array}{cc}
\tilde{G}_{1,0}^{i-1}-\tilde{b}_{i} z^{-\tilde{\beta}_{i}} \tilde{G}_{0,0}^{i-1} & \tilde{G}_{1,1}^{i-1}-\tilde{b}_{i} z^{-\tilde{\beta}_{i}} \tilde{G}_{0,1}^{i-1} \\
\tilde{G}_{0,0}^{i-1} & \tilde{G}_{0,1}^{i-1}
\end{array}\right] \\
& =(-1)^{j_{0}+i-1} \times \\
& {\left[\begin{array}{cc}
-G_{1,0}^{i-1}+b_{i} z^{-\beta_{i}} G_{0,0}^{i-1} & G_{1,1}^{i-1}-b_{i} z^{-\beta_{i}} G_{0,1}^{i-1} \\
G_{0,0}^{i-1} & -G_{0,1}^{i-1}
\end{array}\right]} \\
& =(-1)^{j_{0}+i}\left[\begin{array}{cc}
G_{0,0}^{i} & -G_{0,1}^{i} \\
-G_{1,0}^{i} & G_{1,1}^{i}
\end{array}\right] \text {. }
\end{aligned}
$$

After the decomposition steps, the initialization matrices are obtained

$$
\begin{aligned}
& \boldsymbol{G}_{l, \text { ini }}(z)=\left[\begin{array}{cc}
g_{l, 0} & g_{l, 1} \\
z^{-1} g_{l, 2} & z^{-1} g_{l, 3}
\end{array}\right], \\
& \tilde{\boldsymbol{G}}_{l, \text { ini }}(z)=(-1)^{i_{0}+j_{0}}\left[\begin{array}{cc}
g_{l, 0} & -g_{l, 1} \\
-z^{-1} g_{l, 2} & z^{-1} g_{l, 3}
\end{array}\right] .
\end{aligned}
$$

This can be easily verified by (32) with maximally $i=i_{0}$ possible steps. It can be seen that only some signs in the factorization steps are changed for the SMFB filter bank. Therefore, we only need to calculate the factorization for the CMFB, and the SMFB factorization can then be computed by only a few sign changes.

\section{DC-LEAKAGE-FREE PROPERTY}

In order to obtain a filter bank without DC-leakage, our filter coefficients need to satisfy the following constraints: $\left.H_{k}(z)\right|_{z=1}=\left.\tilde{H}_{k}(z)\right|_{z=1}=0$ and $\left.H_{0}(z)\right|_{z=1}=$ $\left.\tilde{H}_{0}(z)\right|_{z=1}=1$ [8]. From (2), the constraints can be expressed as

$$
\begin{aligned}
& {[1,0, \ldots, 0]^{T}=\left.\boldsymbol{E}\left(z^{M}\right)[1,1, \ldots, 1]^{T}\right|_{z=1},} \\
& {[1,0, \ldots, 0]^{T}=\left.\tilde{\boldsymbol{E}}\left(z^{M}\right)[1,1, \ldots, 1]^{T}\right|_{z=1} .}
\end{aligned}
$$


Furthermore, using (9) and (15) we can write the polyphase matrices $\boldsymbol{E}(z)$ and $\tilde{\boldsymbol{E}}(z)$ as

$$
\begin{aligned}
\boldsymbol{E}(z) & =\tilde{\boldsymbol{C}}_{1} \boldsymbol{G}(z), \\
\tilde{\boldsymbol{E}}(z) & =\tilde{\boldsymbol{S}}_{1} \tilde{\boldsymbol{G}}(z)
\end{aligned}
$$

where

$$
\begin{gathered}
{\left[\tilde{\boldsymbol{C}}_{1}\right]_{k, l}=\left[\boldsymbol{C}_{1}\right]_{k, l}, \quad\left[\tilde{\boldsymbol{C}}_{1}\right]_{k, M-1-l}=\left[\boldsymbol{C}_{1}\right]_{k, 2 M-1-l},} \\
{\left[\tilde{\boldsymbol{S}}_{1}\right]_{k, l}=\left[\boldsymbol{S}_{1}\right]_{k, l}, \quad\left[\tilde{\boldsymbol{S}}_{1}\right]_{k, M-1-l}=\left[\boldsymbol{S}_{1}\right]_{k, 2 M-1-l},} \\
0 \leq k<M, 0 \leq l<M / 2 \\
\boldsymbol{G}_{1}(z)=\operatorname{diag}\left(G_{0}\left(-z^{2}\right), \ldots, G_{M / 2-1}\left(-z^{2}\right),\right. \\
\left.z^{-1} G_{3 M / 2}\left(-z^{2}\right), \ldots, z^{-1} G_{2 M-1}\left(-z^{2}\right)\right), \\
\boldsymbol{G}_{2}(z)=\operatorname{diag}\left(z^{-1} G_{M}\left(-z^{2}\right), \ldots, z^{-1} G_{3 M / 2-1}\left(-z^{2}\right),\right. \\
\left.-G_{M / 2}\left(-z^{2}\right), \ldots,-G_{M-1}\left(-z^{2}\right)\right), \\
\boldsymbol{G}(z)=\boldsymbol{G}_{1}(z)+(-1)^{s-1} \boldsymbol{J G}_{2}(z), \\
\tilde{\boldsymbol{G}}(z)=\boldsymbol{G}_{1}(z)+(-1)^{s} \boldsymbol{J G}_{2}(z) .
\end{gathered}
$$

Equation (34) leads to

$$
\begin{aligned}
\tilde{\boldsymbol{C}}_{1}^{-1}[1,0, \ldots, 0]^{T} & =\left.\boldsymbol{G}\left(z^{M}\right)[1,1, \ldots, 1]^{T}\right|_{z=1}, \\
\tilde{\boldsymbol{S}}_{1}^{-1}[1,0, \ldots, 0]^{T} & =\left.\tilde{\boldsymbol{G}}\left(z^{M}\right)[1,1, \ldots, 1]^{T}\right|_{z=1} .
\end{aligned}
$$

By splitting $\boldsymbol{G}(z)$ and $\tilde{\boldsymbol{G}}(z)$ into the submatrices $\boldsymbol{G}_{l}(z)$ and $\tilde{G}_{l}(z)$ and using (16) and (20), we obtain the constraints as

$$
\begin{aligned}
{\left[\begin{array}{c}
{\left[\tilde{\boldsymbol{C}}_{1}^{-1}\right]_{l, 0}} \\
{\left[\tilde{\boldsymbol{C}}_{1}^{-1}\right]_{M-1-l, 0}}
\end{array}\right]=} & \prod_{j=1}^{j_{0}}\left[\begin{array}{cc}
d_{l, j} & 1 \\
1 & 0
\end{array}\right] \prod_{i=1}^{i_{0}}\left[\begin{array}{ll}
0 & 1 \\
1 & b_{l, i}
\end{array}\right]\left[\begin{array}{l}
g_{l, 0}+g_{l, 1} \\
g_{l, 2}+g_{l, 3}
\end{array}\right] \\
{\left[\begin{array}{c}
{\left[\tilde{\boldsymbol{S}}_{1}^{-1}\right]_{l, 0}} \\
{\left[\tilde{\boldsymbol{S}}_{1}^{-1}\right]_{M-1-l, 0}}
\end{array}\right]=} & \prod_{j=1}^{j_{0}}\left[\begin{array}{cc}
d_{l, j} & -1 \\
-1 & 0
\end{array}\right] \times \\
& \prod_{i=1}^{i_{0}}\left[\begin{array}{cc}
0 & -1 \\
-1 & b_{l, i}
\end{array}\right]\left[\begin{array}{l}
g_{l, 0}-g_{l, 1} \\
g_{l, 3}-g_{l, 2}
\end{array}\right]
\end{aligned}
$$

or, equivalently

$$
\begin{aligned}
{\left[\begin{array}{l}
g_{l, 0}+g_{l, 1} \\
g_{l, 2}+g_{l, 3}
\end{array}\right]=} & \prod_{i=i_{0}}^{1}\left[\begin{array}{cc}
-b_{l, i} & 1 \\
1 & 0
\end{array}\right] \times \\
& \prod_{j=j_{0}}^{1}\left[\begin{array}{cc}
0 & 1 \\
1 & -d_{l, j}
\end{array}\right]\left[\begin{array}{c}
{\left[\tilde{\boldsymbol{C}}_{1}^{-1}\right]_{l, 0}} \\
{\left[\tilde{\boldsymbol{C}}_{1}^{-1}\right]_{M-1-l, 0}}
\end{array}\right], \\
{\left[\begin{array}{l}
g_{l, 0}-g_{l, 1} \\
g_{l, 3}-g_{l, 2}
\end{array}\right]=} & \prod_{i=i_{0}}^{1}\left[\begin{array}{cc}
-b_{l, i} & -1 \\
-1 & 0
\end{array}\right] \times \\
& \prod_{j=j_{0}}^{1}\left[\begin{array}{cc}
0 & -1 \\
-1 & -d_{l, j}
\end{array}\right]\left[\begin{array}{c}
{\left[\tilde{\boldsymbol{S}}_{1}^{-1}\right]_{l, 0}} \\
{\left[\tilde{\boldsymbol{S}}_{1}^{-1}\right]_{M-1-l, 0}}
\end{array}\right] .
\end{aligned}
$$

Eventually, we can verify that designing filter banks without DC leakage is possible by enforcing the constraints given in (37). In addition, we have four different equations with only four different linear unknown variables $g_{l, 0}, g_{l, 1}, g_{l, 2}$ and $g_{l, 3}$ for fixed $b_{l, i}$ and $d_{l, j}$. In other words, our solution for the initialization matrices is unique. This was different for the solely cosine modulated filter banks in [8].
TABLE I

FACTORIZATION OF PROTOTYPE WITH $N=16$ AND $M=4$

\begin{tabular}{cc|cc} 
& $l$ & 0 & 1 \\
\hline \multirow{3}{*}{ Both } & $\delta_{l, 1}$ & 1 & 1 \\
& $d_{l, 1}$ & -0.1263 & -0.0300 \\
& $\beta_{l, 2}$ & 1 & 1 \\
& $b_{l, 2}$ & 0.1244 & 0.0300 \\
\hline \multirow{3}{*}{ DC leakage } & $g_{l, 0}$ & -0.1750 & -0.1495 \\
& $g_{l, 1}$ & 0.0579 & 0.1056 \\
& $g_{l, 2}$ & 0.0570 & 0.1055 \\
& $g_{l, 3}$ & 0.1723 & 0.1493 \\
\hline \multirow{3}{*}{ No DC leakage } & $g_{l, 0}$ & -0.1690 & -0.1440 \\
& $g_{l, 1}$ & 0.0564 & 0.1026 \\
& $g_{l, 2}$ & 0.0555 & 0.1025 \\
& $g_{l, 3}$ & 0.1664 & 0.1439
\end{tabular}

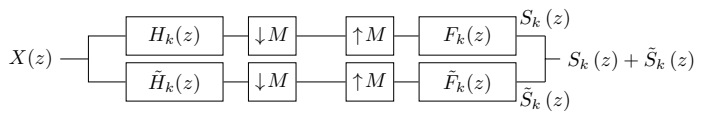

Fig. 3. The k-th subband signal from the first and second system

\section{EXPERIMENTS}

To demonstrate the properties of our approach, we start with a prototype designed according to the method in [10]. The filter optimizes the stopband attenuation as in [9], [10] by minimizing

$$
\sum_{k=0}^{M-1} \int_{\Omega_{s}(k)}\left|H_{k}\left(e^{j \omega}\right)\right|^{2} d \omega,
$$

where $\Omega_{s}(k)$ is the stopband of $H_{k}\left(e^{j \omega}\right)$. The stopbands were set to $\Omega_{s}(k)=\left[0, \max \left\{0, \frac{\pi}{M}(k-2)\right\}\right] \cup$ $\left[\min \left\{\frac{\pi}{M}(k+2), \pi\right\}, \pi\right]$. Because the filter optimization according to [10] does not result in filters that are free from DC-leakage, we used the obtained prototype and calculated the decomposition into maximum-delay, zero-delay, and initialization matrices, and then we enforced the constraints given by (37).

In Fig. 2 and Table I, we show the results for an original, not DC-leakage compensated prototype and its counterpart with the constraints in (37). It can be seen that the passband behavior of the filters remains unchanged when enforcing (37), but the zero at $\omega=0$ is exactly met for all bandpass filters.

In [15], the shift invariance of a system was formulated as (see also Fig. 3)

$$
S_{k, r}(z)+\tilde{S}_{k, r}(z)=z^{-r}\left(S_{k, 0}(z)+\tilde{S}_{k, 0}(z)\right) .
$$

Here, $r$ denotes the shift of the signal, and $S_{k, r}(z)$ and $\tilde{S}_{k, r}(z)$ are the $k$-th subband signals of our CSMFB where the signal is shifted by $r$. Following [15], shift invariance can be measured as:

$$
R_{a}^{k}=\frac{\sum_{l=1}^{M-1} \mathcal{E}\left\{H_{k}\left(W_{M}^{l} z\right) F_{k}(z)+\tilde{H}_{k}\left(W_{M}^{l} z\right) \tilde{F}_{k}(z)\right\}}{\mathcal{E}\left\{H_{k}(z) F_{k}(z)+\tilde{H}_{k}(z) \tilde{F}_{k}(z)\right\}},
$$

where $\mathcal{E}\{U(z)\}$ represents the energy of a filter with impulse response $u(n)$. We represent the values for $R_{k}$ in $\mathrm{dB}$ in Table II. 


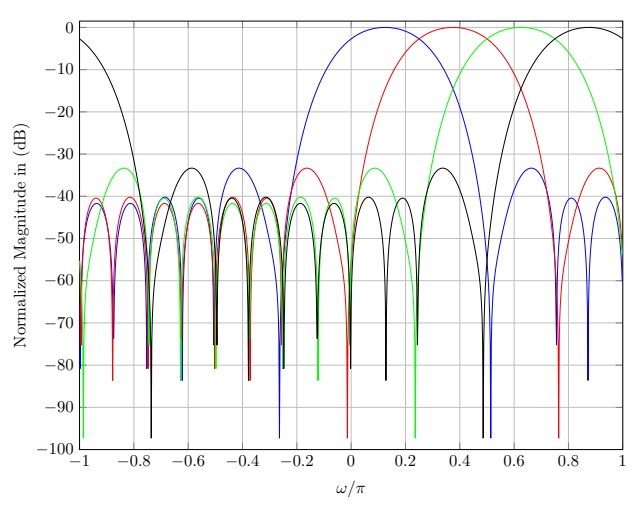

(a) with DC leakage

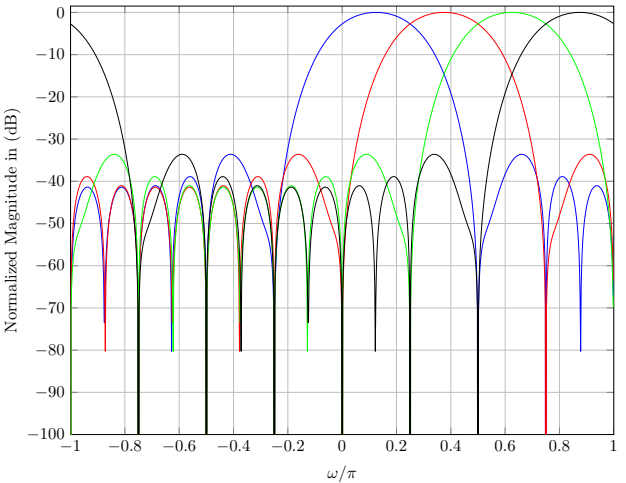

(b) without DC leakage

Fig. 2. Filter bank arising form prototype filter with (left) and without (right) DC leakage; $M=4, N=16$

TABLE II

SHIFT-INVARIANCE $R_{k}$ FOR DIFFERENT PROTOTYPES IN DB

\begin{tabular}{ccc|cccc} 
& & & \multicolumn{4}{|c}{$k$} \\
DC-leakage & $M$ & $N$ & 0 & 1 & 2 & 3 \\
\hline $\mathrm{x}$ & 4 & 16 & -31.3464 & -26.9509 & -26.9509 & -31.3464 \\
& 4 & 16 & -31.7250 & -27.2442 & -27.2442 & -31.7250 \\
$\mathrm{x}$ & 4 & 32 & -49.6990 & -44.3638 & -44.3638 & -49.6990 \\
& 4 & 32 & -49.6646 & -44.3194 & -44.3194 & -49.6646 \\
$\mathrm{x}$ & 8 & 32 & -30.9160 & -26.4100 & -26.2835 & -26.2676 \\
& 8 & 32 & -31.2742 & -26.6775 & -26.5069 & -26.4890 \\
$\mathrm{x}$ & 8 & 64 & -38.3457 & -34.0223 & -34.1355 & -34.1219 \\
& 8 & 64 & -37.0661 & -33.0135 & -33.2360 & -33.2252
\end{tabular}

For prototypes with shorter lengths, enforcing the constraints in (37) produces better shift-invariance as shown in Table II. In case of longer prototypes, the shift-invariance become worse by enforcing the constraints, but the difference is insignificant.

\section{CONCLUSION}

We introduced a new structure for CSMFB in which the prototype is easily enforced with the constraints to result in a filter bank without DC-leakage. We showed that by enforcing the constraints, the passband behavior of a prototype is not significantly changed. In addition, in the optimization step we can directly obtain the prototypes for CSMBF without DCleakage only by setting the initialization matrices depending on the free parameters $b_{l}$ and $d_{l}$. We expect that directly optimizing on the free parameters of the filter bank would result in better CSMFB prototypes. Finally, it should be noted that the proposed lifting structure allows a CSMFB implementation with a minimal number of operations required.

\section{ACKNOWLEDGMENT}

This work was supported by the Graduate School for Computing in Medicine and Life Sciences, funded by the German Federal Government's Excellence Initiative (grant number DFG GSC 235/1)

\section{REFERENCES}

[1] I. W. Selesnick, R. G. Baraniuk, and N. G. Kingsbury, "The dual-tree complex wavelet transform," IEEE Signal Processing Magazine, vol. 22, no. 6, pp. 123-151, Nov. 2005
[2] C. Chaux, L. Duval, and J.-C. Pesquet, "Image analysis using a dual-tree m-band wavelet transform," IEEE Transactions on Image Processing, vol. 15, no. 8, pp. 2397-2412, Aug. 2006.

[3] I. Bayram and I. W. Selesnick, "On the dual-tree complex wavelet packet and $\mathrm{m}$-band transforms," IEEE Transactions on Signal Processing, vol. 56, no. 6, pp. 2298-2310, Jun. 2008.

[4] S. Kyochi, T. Uto, and M. Ikehara, "Dual-tree complex wavelet transform arising from cosine-sine modulated filter banks," in IEEE International Symposium on Circuits and Systems, May 2009, pp. 2189-2192.

[5] A. Viholainen, T. Stitz, J. Alhava, T. Ihalainen, and M. Renfors, "Complex modulated critically sampled filter banks based on cosine and sine modulation," in IEEE International Symposium on Circuits and Systems, vol. 1, May 2002, pp. I-833-I-836.

[6] S. Kyochi and M. Ikehara, "A class of near shift-invariant and orientation-selective transform based on delay-less oversampled evenstacked cosine-modulated filter banks," IEICE Transactions on Fundamentals of Electronics, Communications and Computer Sciences, vol. 93-A, no. 4, pp. 724-733, Apr. 2010.

[7] L. Liang and H. Liu, "Dual-tree cosine-modulated filter bank with linearphase individual filters: An alternative shift-invariant and directionalselective transform," IEEE Transactions on Image Processing, vol. 22, no. 12, pp. 5168-5180, Dec. 2013.

[8] T. Karp, A. Mertins, and G. Schuller, "Efficient biorthogonal cosinemodulated filter banks," Signal Processing, vol. 81, no. 5, pp. 997-1016, May 2001.

[9] S. Kyochi, T. Suzuki, and Y. Tanaka, "A directional and shift-invariant transform based on m-channel rational-valued cosine-sine modulated filter banks," in Asia-Pacific Signal Information Processing Association Annual Summit and Conference, Dec. 2012, pp. 1-4.

[10] — "A two-dimensional non-separable implementation of dyadicvalued cosine-sine modulated filter banks for low computational complexity," in Asia-Pacific Signal Information Processing Association Annual Summit and Conference, Oct. 2013, pp. 1-6.

[11] A. Viholainen, J. Alhava, and M. Renfors, "Implementation of parallel cosine and sine modulated filter banks for equalized transmultiplexer systems," in IEEE International Conference on Acoustics, Speech, and Signal Processing, vol. 6, May 2001, pp. 3625-3628.

[12] - "Efficient implementation of complex modulated filter banks using cosine and sine modulated filter banks," EURASIP Journal on Advances in Signal Processing, vol. 2006, pp. 1-10, Apr. 2006.

[13] H. S. Malvar, "Extended lapped transforms: properties, applications, and fast algorithms," IEEE Transactions on Signal Processing, vol. 40, no. 11, pp. 2703-2714, Nov. 1992.

[14] P. N. Heller, T. Karp, and T. Q. Nguyen, "A general formulation of modulated filter banks," IEEE Transactions on Signal Processing, vol. 47, no. 4, pp. 986-1002, Apr. 1999.

[15] N. Kingsbury, "Complex wavelets for shift invariant analysis and filtering of signals," Applied and Computational Harmonic Analysis, vol. 10, no. 3, pp. 234-253, May 2001. 